\title{
Head-on beam-beam compensation investigation in an electron-ion collider using weak-strong simulations
}

\author{
Christoph Montag and Wolfram Fischer \\ Brookhaven National Laboratory, Upton, New York 11973, USA \\ (Received 17 October 2008; published 19 August 2009)
}

\begin{abstract}
The luminosity of the ring-ring version of the proposed electron-ion collider eRHIC is limited by the beam-beam effect on the electrons. Once the beam-beam limit is reached, the luminosity no longer increases linearly with the bunch intensity of the ion beam, but begins to saturate and even drops again if the beam-beam tuneshift is increased further. To overcome this limitation we investigate a compensation scheme with an electron lens acting on the electron beam. Using weak-strong simulations we find a possible luminosity increase of about a factor 2 .
\end{abstract}

DOI: 10.1103/PhysRevSTAB.12.084001

PACS numbers: $29.20 . \mathrm{db}, 29.27 . \mathrm{Bd}$

\section{INTRODUCTION}

The beam-beam effect experienced by the electron beam in the proposed circular electron-ion collider eRHIC [1] is a major obstacle in increasing the luminosity of this facility. When the ion bunch intensity is increased in an effort to maximize the luminosity, the nonlinear beam-beam interaction results in two effects on the electron beam when the beam-beam limit is approached.

The first of these effects is an increase in the core emittance of the electron beam, causing a beam size increase at the interaction point. However, unless this emittance blowup is too large, this effect can be partially compensated for by a proper choice of the working point of the electron ring, resulting in dynamic focusing at the interaction point (IP).

The second beam-beam limit is characterized by the development of a non-Gaussian halo of the electron beam. Since this halo needs to be accommodated in the aperture of the machine to ensure sufficient electron beam lifetime, the aperture limitation in the low- $\beta$ quadrupoles around the IP provides a limit on the attainable ion bunch intensity $N_{p}$ and therefore the luminosity.

Compensation of the nonlinear beam-beam kick in storage rings by means of an electron lens located in a second interaction region was proposed by Talman in 1976 [2], following the failure of a four-beam compensation scheme in DCI [3] due to coherent instabilities [4,5]. In 1993, Tsyganov et al. studied the feasibility of beam-beam compensation in proton-proton colliders [6,7]. In the late 1990s, Shiltsev et al. proposed and built an electron lens to compensate small bunch-to-bunch tune variations caused by long-range beam-beam effects in the Tevatron [8-12]. The applicability of head-on beam-beam compensation in RHIC and LHC is under study in both simulations [13-15] and hardware design considerations.

For ideal beam-beam compensation, three conditions need to be fulfilled: (i) linear transport between IP and electron lens; (ii) betatron phase advance of $k \cdot 180^{\circ}$ be- tween IP and electron lens, where $k$ is an integer; (iii) same amplitude-dependent kick, but with opposite sign.

In this paper, we investigate the feasibility of such a scheme to increase the beam-beam limit in the electron ring of an electron-ion collider. We assume throughout the rest of the paper that the beam-beam effect of the electron beam on the ion beam does not limit the luminosity, i.e., we are using a weak-strong model in which the electron beam is weak and the ion beam strong.

Our case differs from the head-on beam-beam compensation scheme in DCI. In DCI every beam could couple back to the three other beams via the beam-beam interaction. In our case the electron beam of the electron lens does not couple back to the electron beam of the electron ring except for single pass effects. We therefore do not expect the coherent effects observed in DCI [3] and studied in simulations later $[16,17]$ to be present in the compensation scheme proposed here.

Our case also differs from head-on beam-beam compensation schemes with electron lenses studied for hadron beams because the electron beam is damped through the synchrotron light emission. For hadron beams the possibility of coherent beam centroid motion was raised that would result in emittance growth [18], in particular under conditions with head-on beam-beam compensation [19]. However, although coherent effects have been observed in both the Tevatron [20] and RHIC [21], the performance of neither machine is limited by such an effect to date. In Ref. [22] the possibility of coherent effects in eRHIC was raised although with a linear lattice model only. Whether strong-strong simulations will be needed is therefore an open question.

We perform tracking studies with a simplified model of the electron storage ring, the main parameters of which are taken from the eRHIC ring-ring collider design [1]. While the eRHIC design assumes a proton bunch intensity of $N_{p}=1 \times 10^{11}$, corresponding to a vertical beam-beam tuneshift parameter of $\xi_{y}=0.08$ of the electron beam, we increase the beam-beam parameter here by increasing 
the proton bunch intensity $N_{p}$ beyond that value. The same effect could be achieved by assuming smaller emittances. To keep the beam sizes matched, the emittance of both beams could be reduced while keeping the $\beta$ functions unchanged.

In the course of this paper, we first describe the electron ring model. Then we calculate luminosities over a range of tunes for partial and full beam-beam compensation with a beam-beam parameter larger than the largest beam-beam parameters achieved today. Next, we investigate the effect of the electron lens on the non-Gaussian tails over a range of electron beam-beam parameters, and study the robustness of the beam-beam compensation scheme with respect to variations of some of the electron lens beam parameters. Finally, we use all previously gained information to maximize the luminosity with an electron lens.

\section{THE ELECTRON RING MODEL}

The electron ring model used for the simulation studies presented in this paper is comprised of $N_{\mathrm{FODO}}=50$ identical FODO cells, equipped with sextupoles for chromaticity correction. The electron-proton interaction point (IP) and the electron lens are separated by $N_{\text {sep }}$ FODO cells, which form one arc. The other arc of the machine therefore consists of $N_{\text {FODO }}-N_{\text {sep }}$ FODO cells, as schematically illustrated in Fig. 1. At both ends of each arc, an ideal dispersion suppressor is located, resulting in both zero dispersion $D^{*}$ and zero derivative $D^{* \prime}=d D^{*} / d s$ at the IP and the electron lens. Low- $\beta$ focusing in the two transverse planes

$$
\begin{aligned}
\vec{x} & =\left(\begin{array}{c}
x \\
x^{\prime}
\end{array}\right), \\
\vec{y} & =\left(\begin{array}{c}
y \\
y^{\prime}
\end{array}\right)
\end{aligned}
$$

at the IP and the electron lens is realized by chromatic matrices of the form

$$
M_{\text {telescope }}=\left(\begin{array}{cc}
\sqrt{\frac{\beta^{*}}{\beta_{\text {arc }}}} \cos \phi & \sqrt{\beta^{*} \beta_{\text {arc }}} \sin \phi \\
-\frac{1}{\sqrt{\beta^{*} \beta_{\text {arc }}}} \sin \phi & \sqrt{\frac{\beta_{\text {arc }} \beta^{*}}{2}} \cos \phi
\end{array}\right),
$$

with $\phi=Q_{\text {telescope }}^{\prime} \cdot \frac{\Delta p}{p}$, where $Q_{\text {telescope }}^{\prime}$ is the chromaticity of the low- $\beta$ telescope. Quantum excitation and synchrotron radiation damping are implemented at the end of each of the two arcs, taking into account the length difference (different number of FODO cells) accordingly. Together with the chromaticity correction sextupoles in each FODO cell, this arrangement ensures realistic nonlinearity and stochasticity between the IP and the electron lens. Table I lists the parameters of the electron ring model.

The presence of the beam-beam lenses at the IP and the electron lens modifies the accelerator lattice. The effect on the linear lattice, which can be described in terms of the
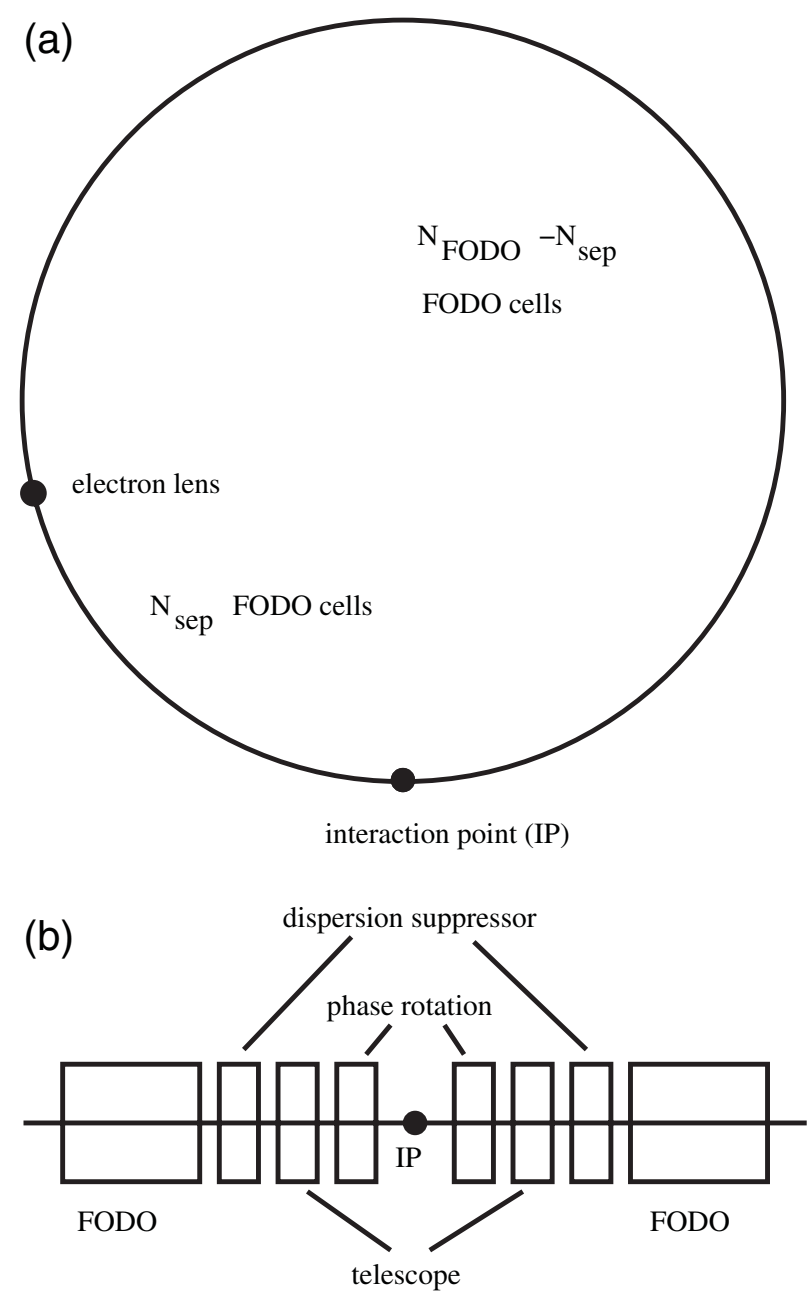

FIG. 1. Schematic drawings of the model electron ring (top) with interaction point (IP) and electron lens connected by two arcs consisting of $N_{\text {sep }}$ and $N_{\text {FODO }}-N_{\text {sep }}$ identical FODO cells. The bottom figure depicts a low- $\beta$ insertion with dispersion suppressors, low- $\beta$ telescopes, and phase-rotation matrices.

Twiss parameters, can be calculated by taking into account the linearized beam-beam kicks at these two locations. The one-turn matrices $M_{\mathrm{IP}}^{\circ}$ and $M_{\text {elens }}^{\circ}$ describing the linear lattice with respect to the IP and the electron lens as reference points can be written as

$$
\begin{gathered}
M_{\mathrm{IP}}^{\circ}=M_{\mathrm{IP} \leftarrow \text { elens }} \cdot M_{\text {elens }} \cdot M_{\text {elens } \leftarrow \mathrm{IP}} \cdot M_{\mathrm{IP}}, \\
M_{\text {elens }}^{\circ}=M_{\text {elens } \leftarrow \mathrm{IP}} \cdot M_{\mathrm{IP}} \cdot M_{\mathrm{IP} \leftarrow \text { elens }} \cdot M_{\text {elens }},
\end{gathered}
$$

where $M_{\mathrm{IP} \leftarrow \text { elens }}$ and $M_{\text {elens } \leftarrow \mathrm{IP}}$ describe the linear lattices of the two arcs between the IP and the electron lens, while $M_{\mathrm{IP}}$ and $M_{\text {elens }}$ are thin lenses of the form

$$
M=\left(\begin{array}{cc}
1 & 0 \\
\frac{1}{f} & 1
\end{array}\right),
$$

describing the linearized beam-beam kick. The focal lengths $f$ of these two matrices are expressed by the $\beta$ 
TABLE I. Parameter list of the accelerator model. $\beta$ functions and rms beam sizes at the IP and electron lens, as well as damping times, are taken from the eRHIC ring-ring design [1], while the proton beam intensity $N_{p}$ is quadrupled.

\begin{tabular}{lcc}
\hline \hline Number of FODO cells & $N_{\text {FODO }}$ & 50 \\
Number of cells between IP and electron lens & $N_{\text {sep }}$ & 10 \\
Phase advance/cell (horizontal/vertical) & $\Delta \Phi_{x} / \Delta \Phi_{y}$ & $79.7^{\circ} / 89.0^{\circ}$ \\
Chromaticity (horizontal/vertical) & $Q_{x, y}^{\prime}=\Delta Q_{x, y} / \frac{\Delta p}{p}$ & $+2 /+2$ \\
Synchrotron tune & $Q_{s}$ & 0.015 \\
rms bunch length & $\sigma_{s}$ & $0.0117 \mathrm{~m}$ \\
rms momentum spread & $\sigma_{p}$ & $9.4 \times 10^{-4}$ \\
$\beta$ function at IP and electron lens & $\beta_{x} / \beta_{y}$ & $0.19 \mathrm{~m}, 0.26 \mathrm{~m}$ \\
Number of positive charges/bunch & $N_{p}$ & $4 \times 10^{11}$ \\
Electron lens intensity/bunch & $N_{e}$ & $4 \times 10^{11}$ \\
rms proton beam size at IP & $\sigma_{x, p} / \sigma_{y, p}$ & $101 \mu \mathrm{m} / 50.5 \mu \mathrm{m}$ \\
rms electron lens beam size & $\sigma_{x, e} / \sigma_{y, e}$ & $101 \mu \mathrm{m} / 50.5 \mu \mathrm{m}$ \\
Lorentz factor & $\gamma$ & 19560 \\
Electron beam-beam parameter & $\xi_{x} / \xi_{y}$ & $0.11 / 0.32$ \\
Damping times & $\tau_{x} / \tau_{y} / \tau_{z}$ & $1740 / 1740 / 870$ turns \\
\hline \hline
\end{tabular}

function and the respective beam-beam tuneshift parameter $\xi$ of the IP and the electron lens as

$$
f=\frac{\beta}{4 \pi \xi},
$$

with the beam-beam parameter given as

$$
\xi_{x, y}=\frac{N_{e, p} \beta_{x, y} r_{e}}{2 \pi \gamma \sigma_{x, y}\left(\sigma_{x}+\sigma_{y}\right)} .
$$

In this equation, $N_{e, p}, \sigma_{x}$, and $\sigma_{y}$ denote the bunch intensity and horizontal and vertical beam size of the oncoming electron lens (subscript $e$ ) or proton (subscript $p$ ) beam, respectively. $\gamma$ is the Lorentz factor of the stored electron beam, and $\beta_{x, y}$ are its horizontal and vertical $\beta$ functions at the IP and the electron lens, while $r_{e}$ is the classical

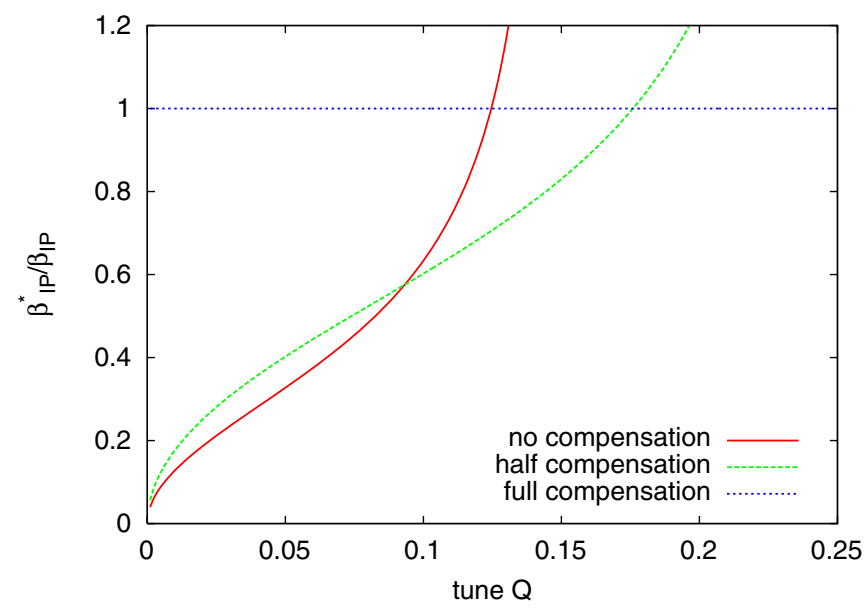

FIG. 2. (Color) Dynamic $\beta$ functions at the IP, for full $\left(N_{e}=\right.$ $\left.N_{p}\right)$, half $\left(N_{e}=0.5 \cdot N_{p}\right)$, and no $\left(N_{e}=0\right)$ beam-beam compensation, for a beam-beam parameter $\xi=0.32$, corresponding to $N_{p}=4 \times 10^{11}$. electron radius. It is worth mentioning here that the beam-beam parameters at the IP and at the electron lens have opposite signs-positive at the IP, negative at the electron lens.

The resulting betatron tunes $Q^{*}$ are computed using the trace of either resulting one-turn matrix,

$$
\cos \left(2 \pi Q^{*}\right)=\frac{1}{2} \operatorname{Tr}\left(M_{\mathrm{IP}}^{\circ}\right)=\frac{1}{2} \operatorname{Tr}\left(M_{\text {elens }}^{\circ}\right),
$$

while the $\beta$ functions at the IP and the electron lens, $\beta_{\mathrm{IP}}^{*}$ and $\beta_{\text {elens }}^{*}$, are calculated from the matrix elements $M_{12}^{\circ}$ of the corresponding one-turn matrices $M_{\mathrm{IP}}^{\circ}$ and $M_{\text {elens }}^{\circ}$ and the resulting tune $Q^{*}$ as

$$
\begin{gathered}
\beta_{\mathrm{IP}}^{*}=\left.M_{\mathrm{IP}}^{\circ}\right|_{12} / \sin \left(2 \pi Q^{*}\right), \\
\beta_{\text {elens }}^{*}=\left.M_{\text {elens }}^{\circ}\right|_{12} / \sin \left(2 \pi Q^{*}\right) .
\end{gathered}
$$

In the uncompensated or partially compensated case, dynamic focusing leads to a significant reduction of both $\beta_{\mathrm{IP}}^{*}$ and the beam-beam tuneshift $\left|Q^{*}-Q\right|<\xi$ for tunes just above the integer or half-integer resonance. With full headon beam-beam compensation via the electron lens, and a betatron phase advance between IP and electron lens of $m$. $180^{\circ}$, where $m$ is an integer, the dynamic focusing effects cancel at both the IP and the electron lens, as shown in Fig. 2. For the remainder of this paper we limit our tune scans to the range between the integer and the quarter resonance, $0.0<Q_{x, y}<0.25$.

\section{LUMINOSITY AS A FUNCTION OF TUNES}

To determine the luminosity as a function of the working point, 100 particles are tracked over 10000 turns, using a 6D element-by-element tracking technique. The nonlinear beam-beam kicks at the IP and the electron lens are implemented using the complex error function representation [23]. The number of turns, which corresponds to 5.75 
transverse damping times, has been chosen to ensure that the equilibrium beam size is reached with an accuracy well below $1 \%$, while at the same time limiting the required CPU time. The equilibrium rms beam sizes $\sigma_{x, e}$ and $\sigma_{y, e}$ of the electron beam are determined by averaging over the last 1740 turns (one transverse damping time), resulting in a relative error of the equilibrium beam sizes of $1.7 \times$ $10^{-3}$

Using the nominal proton beam sizes $\sigma_{x, p}, \sigma_{y, p}$ and the equilibrium electron beam sizes $\sigma_{x, e}, \sigma_{y, e}$, we define the geometric luminosity factor as

$$
\begin{aligned}
F_{\text {geom }} & =\frac{L}{L_{0}} \\
& =\frac{2 \sigma_{x, p} \sigma_{y, p}}{\sqrt{\left(\sigma_{x, p}^{2}+\sigma_{x, e}^{2}\right)\left(\sigma_{y, p}^{2}+\sigma_{y, e}^{2}\right)}},
\end{aligned}
$$

where $L_{0}$ denotes the luminosity resulting from equal beam sizes for both beams, $\sigma_{x, e}=\sigma_{x, p}, \sigma_{y, e}=\sigma_{y, p} . F_{\text {geom }}$ is a measure of the electron beam core size increase due to the beam-beam interaction. In the absence of any beam-beam effects, $F_{\text {geom }}=1$ unless the beam size is affected or beam
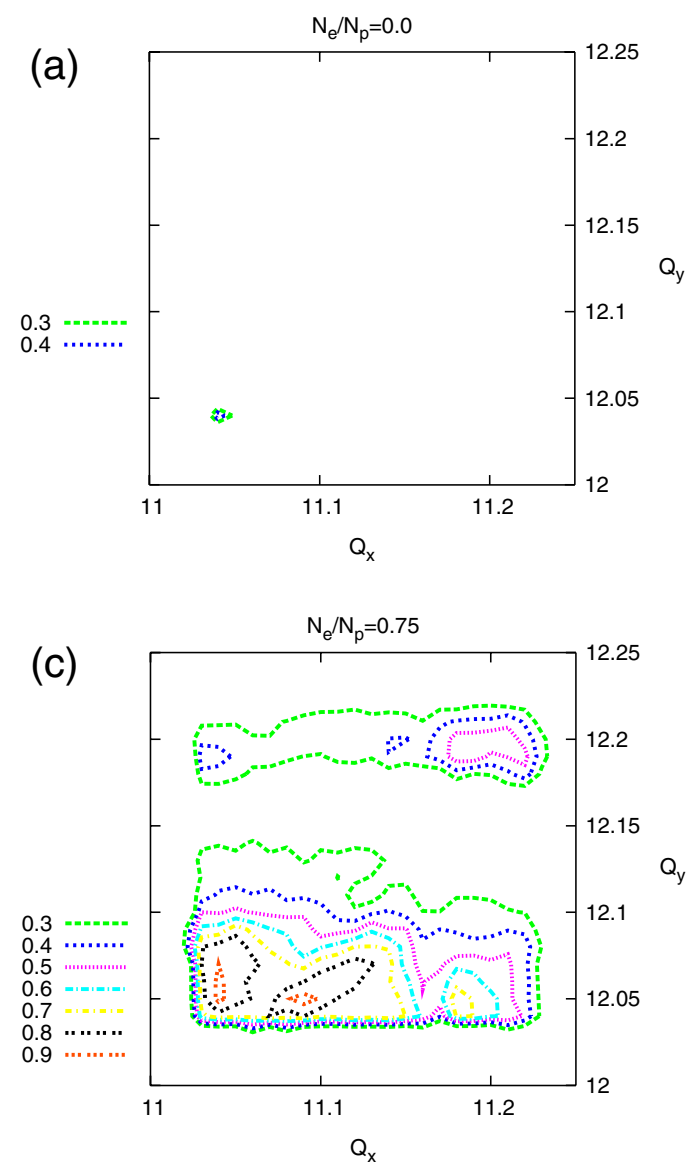

dynamics is unstable due to (nonlinear) lattice resonances. Tracking with $N_{p}=N_{e}=0$ confirmed that $F_{\text {geom }}=1$ for all fractional tunes $0.0<Q_{x, y}<0.25$ besides the integer resonances $Q_{x}=0.0$ or $Q_{y}=0.0$, and the nonlinear coupling resonance $Q_{x}=2 \cdot Q_{y}$. Additionally, we define the normalized luminosity as the product of the geometric luminosity and the proton bunch intensity,

$$
L_{\text {norm }}=F_{\text {geom }} \cdot N_{p} \text {. }
$$

$L_{\text {norm }}$ is proportional to the luminosity for constant electron bunch intensity and constant collision frequency; it is this quantity we aim to maximize.

To determine the optimum working point for the different scenarios, tune scans are performed in steps of $\Delta Q_{x, y}=$ 0.01 . For this purpose the betatron tunes are adjusted by appropriate phase-rotation matrices of the general form,

$$
M_{\mathrm{rot}}=\left(\begin{array}{cc}
\cos (\Delta \phi) & \beta \sin (\Delta \phi) \\
-\frac{\sin (\Delta \phi)}{\beta} & \cos (\Delta \phi)
\end{array}\right)
$$

which are introduced in the dispersion-free region between the low- $\beta$ telescope and the interaction point, as schematically shown in Fig. 1. The advantage of this method in tracking is that it does not require matching of the lattice
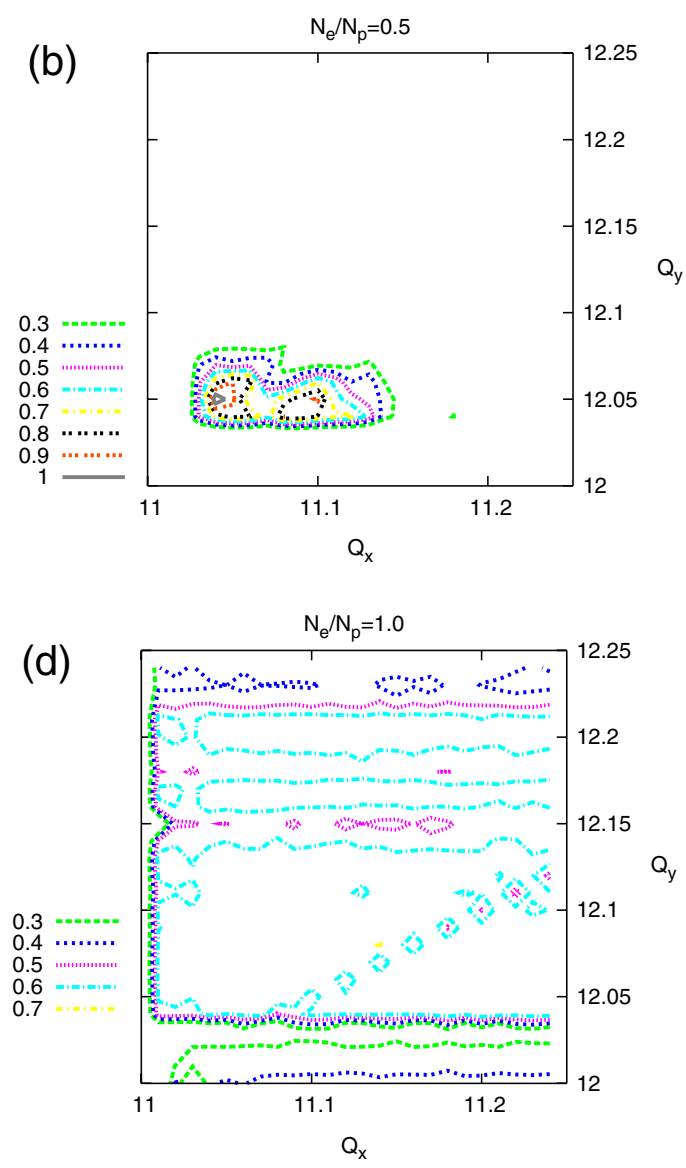

FIG. 3. (Color) Geometric luminosity factor $F_{\text {geom }}$ as a function of tunes with different degrees of head-on beam-beam compensation, with beam and machine parameters as shown in Table I. 
for each individual working point, while leaving the $\beta$ functions at the IP and the electron lens undisturbed. The latter can easily be shown by multiplying the rotation matrix with the linear matrix describing one of the arcs of the machine, for instance $M_{\mathrm{IP} \text { elens }}$ :

$$
\begin{aligned}
& M=M_{\mathrm{rot}} \cdot M_{\mathrm{IP} \leftarrow \text { elens }} \\
& =\left(\begin{array}{cc}
\cos (\Delta \phi) & \beta \sin (\Delta \phi) \\
-\frac{1}{\beta} \sin (\Delta \phi) & \cos (\Delta \phi)
\end{array}\right) \\
& \times\left(\begin{array}{cc}
\cos \phi_{\mathrm{IP} \text { elens }} & \beta \sin \phi_{\mathrm{IP} \leftarrow \text { elens }} \\
-\frac{1}{\beta} \sin \phi_{\mathrm{IP} \text { elens }} & \cos \phi_{\mathrm{IP} \text { elens }}
\end{array}\right) \\
& =\left(\begin{array}{cc}
\cos \left(\phi_{\mathrm{IP} \leftarrow \text { elens }}+\Delta \phi\right) & \beta \sin \left(\phi_{\mathrm{IP} \leftarrow \text { elens }}+\Delta \phi\right) \\
-\frac{\sin \left(\phi_{\mathrm{IP} \leftarrow e l e n s}+\Delta \phi\right)}{\beta} & \cos \left(\phi_{\mathrm{IP} \text { elens }}+\Delta \phi\right)
\end{array}\right) .
\end{aligned}
$$

Here, $\phi_{\mathrm{IP} \leftarrow \text { elens }}$ denotes the betatron phase advance of the arc between electron lens and IP, while $\beta$ is the $\beta$ function at the IP and the electron lens, which are equal.

As a baseline, Fig. 3 shows contour plots of the geometric luminosity factor $F_{\text {geom }}$ for tunes below the quarter resonance, using the parameters listed in Table I. Note that the electron beam-beam parameters are larger than what has been demonstrated in existing machines. Without beam-beam compensation the maximum geometric luminosity factor remains below 0.5 , while with full beambeam compensation, $N_{e}=N_{p}$, it reaches almost 0.7 . Furthermore, beam-beam compensation greatly reduces the tune dependence of the luminosity. While in the absence of compensation the maximum luminosity is attained only in a tiny spot in the tune diagram at $(0.04,0.04)$, a wide tune space provides geometric luminosities above 0.65 in the fully compensated case.

\section{TRANSVERSE TAILS}

Development of long, non-Gaussian transverse tails is often referred to as the second beam-beam limit in $e^{+} e^{-}$ colliders $[24,25]$. A simulation code has been developed to study the transverse electron distribution. This code uses techniques developed by Shatilov [26] and Chen et al. [27], and was benchmarked by reproducing the results given in Ref. [27]. A detailed description of the simulation techniques is given in Ref. [26].

For a Gaussian beam, the distribution density is given as [26]

$$
\rho\left(A_{x}, A_{y}\right)=A_{x} A_{y} \exp \left(-\frac{A_{x}^{2}+A_{y}^{2}}{2}\right) ;
$$

the maximum density is therefore achieved at the point $\left(A_{x}, A_{y}\right)=(1,1)$. Figure 4 shows the corresponding contour lines for $N_{p}=N_{e}=0$, as obtained with the tracking code.

As Fig. 5 shows, the beam dynamics is dominated by the beam-beam limit in the uncompensated case already at a

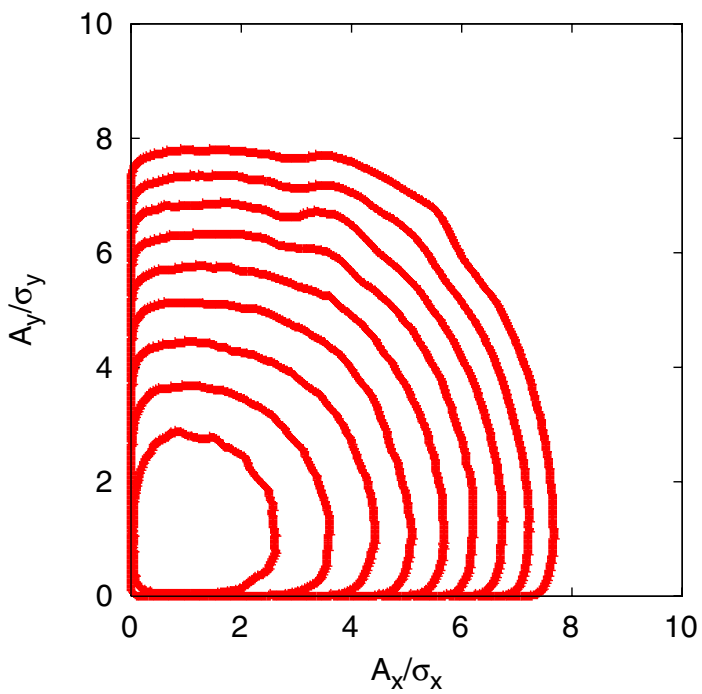

FIG. 4. (Color) Transverse electron distributions without beambeam interaction $\left(N_{p}=N_{e}=0\right)$. The contour lines are spaced by a factor 10 .

proton bunch intensity of $N_{p}=1 \times 10^{11}$, which is the eRHIC ring-ring design intensity [1]. With full electron lens beam-beam compensation, the non-Gaussian tails are almost completely suppressed up to a proton bunch intensity of $N_{p}=2 \times 10^{11}$. At $N_{p}=3 \times 10^{11}$, the transverse tails in the fully compensated case are similar to those obtained in the uncompensated case for $N_{p}=1 \times 10^{11}$, while the fully compensated case for $N_{p}=4 \times 10^{11}$ shows a similar tail distribution as the uncompensated case for $N_{p}=2 \times 10^{11}$. Without beam-beam compensation, transverse tails become extremely pronounced at $N_{p}=$ $3 \times 10^{11}$.

\section{IMPERFECTIONS}

In any real-world application of an electron lens, certain imperfections will lead to less-than-ideal operating conditions. These imperfections include machine errors such as spurious dispersion and beam offsets at the electron lens and the IP, deviations of the betatron phase advance from the ideal value of $m \cdot 180^{\circ}$, and bunch-to-bunch variations in the corresponding proton beam that will lead to a mismatch between the electron lens and the proton beam. The beam-beam compensation scheme needs to be sufficiently robust against unavoidable deviations from the ideal case to ensure a stable operation. It should be emphasized here that this robustness needs to be ensured at a fixed working point, because the intensity and beam size of the oncoming proton bunch vary on a bunch-by-bunch basis, while all electron bunches have the same tune. We investigate the robustness with respect to spurious dispersion, transverse beam offsets, the phase advance between IP and electron lens, intensity mismatch between the proton beam and the electron lens, beam size mismatch due to variations in the 
no compensation, $N_{p}=1 \times 10^{11}$ no compensation, $N_{p}=2 \times 10^{11}$ no compensation, $N_{p}=3 \times 10^{11}$
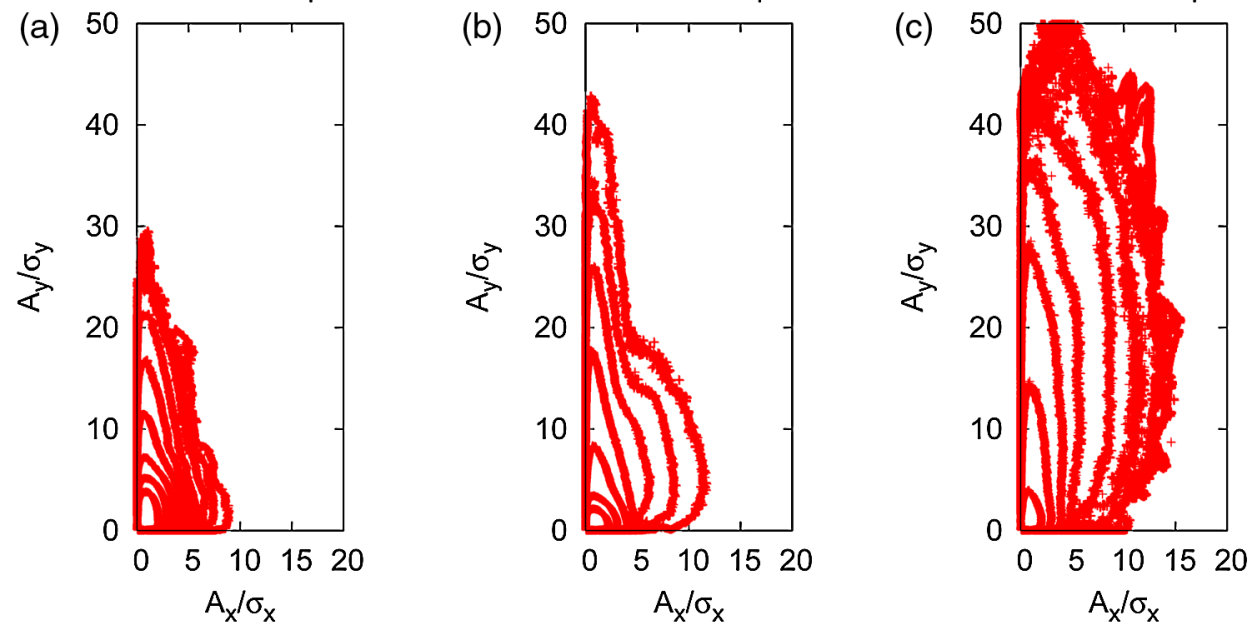

no compensation, $\mathrm{N}_{\mathrm{p}}=4 \times 10^{11}$

half compensation, $N_{p}=1 \times 10^{11}$ half compensation, $N_{p}=2 \times 10^{11}$ half compensation, $N_{p}=3 \times 10^{11}$
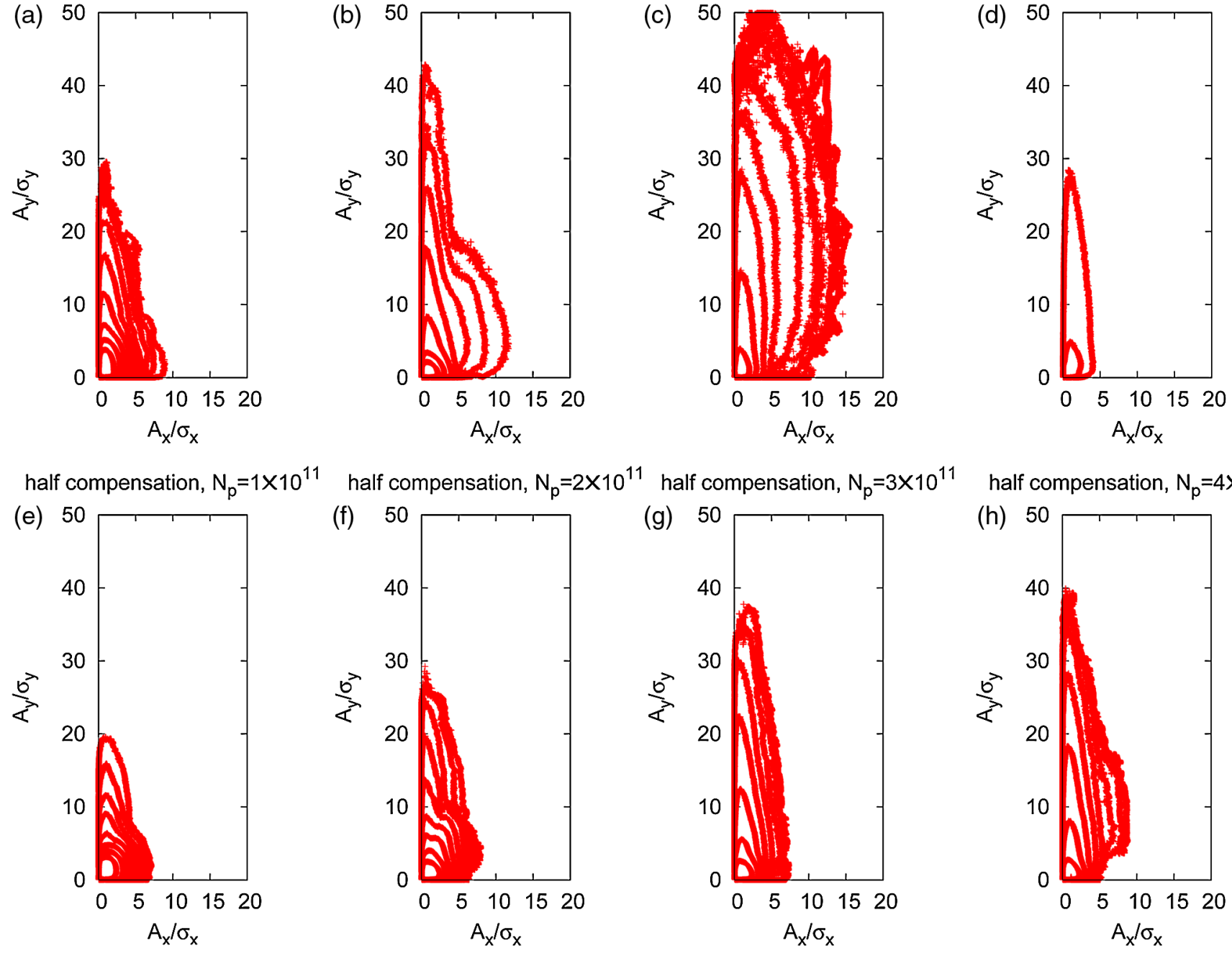

half compensation, $N_{p}=4 \times 10^{11}$

full compensation, $\mathrm{N}_{\mathrm{p}}=1 \times 10^{11}$
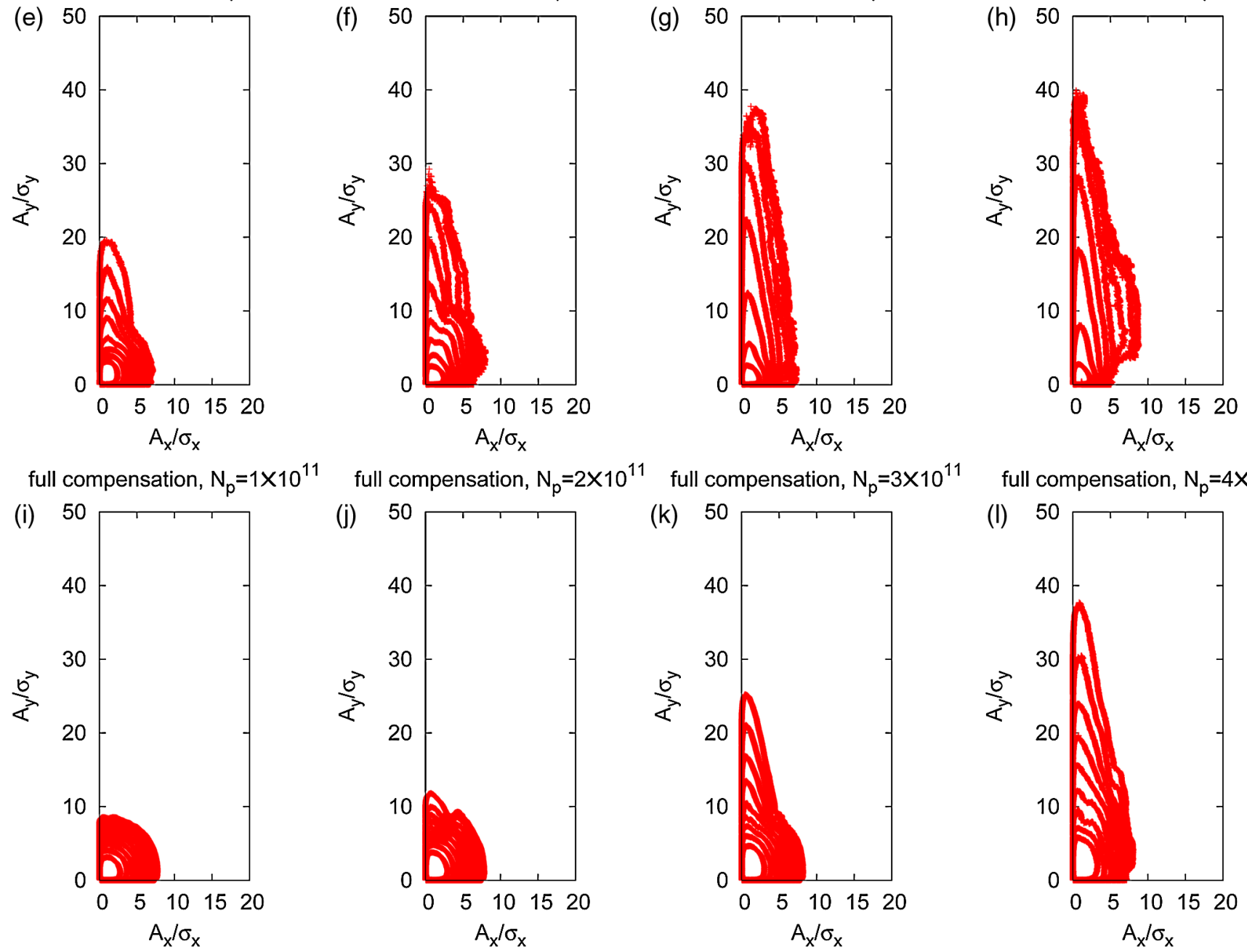

full compensation, $\mathrm{N}_{\mathrm{p}}=4 \times 10^{11}$

FIG. 5. (Color) Transverse electron distributions for uncompensated $\left(N_{e}=0\right)$, half $\left(N_{e} / N_{p}=0.5\right)$, and fully ( $\left.N_{e} / N_{p}=1.0\right)$ compensated cases, for proton bunch intensities $N_{p}$ ranging from $N_{p}=1 \times 10^{11}$ to $N_{p}=4 \times 10^{11}$. In the uncompensated case with $N_{p}=4 \times 10^{11}$, only two contour lines are shown since any further lines are outside the scale. All tail studies were performed at those tunes that, according to tune scans, resulted in the highest luminosity for each particular case. All these tunes are in the vicinity of $(0.05 / 0.06)$. The contour lines are spaced by a factor 10 . 
proton beam size, and nonlinearities between the IP and the electron lens.

For this purpose we perform tune scans over the same fractional tune range $0.0<Q_{x, y}<0.25$ as before, again in steps of $\Delta Q_{x, y}=0.01$. Using the five highest luminosity values determined during the scan, we calculate the average and rms distribution of these five values. The latter is a measure of the attainability of the calculated luminosity in a real machine. For instance, if the highest luminosity is obtained only for one specific working point, while all other working points result in much smaller luminosities, the rms spread becomes comparably large. On the other hand, if the best five (or more) working points deliver similar luminosities, the rms spread becomes very small. In Figs. 7-12, we depicted the spread of luminosities for a given configuration in the form of error bars. Likewise, we assigned "errors" to the luminosity values given in Tables II and III. However, the reader should be aware that these "error bars" are not error bars in the regular sense.

To avoid distorting the luminosity results by simple geometric factors, we introduced all these distortions at the electron lens only, while keeping ideal conditions at the IP. This ensures that any effects on the electron beam size at the IP are results of imperfect beam-beam compensation rather than, for instance, due to coupling of the longitudinal phase space into the transverse plane via spurious dispersion.

\section{A. Spurious dispersion}

To avoid synchrobetatron resonances, one aims at zero horizontal and vertical dispersion $D_{x}, D_{y}$ at both the interaction point and the electron lens. However, in a real machine, this is only approximately the case due to machine errors such as magnet misalignments and quadrupole field errors. In this case, additional synchrobetatron resonances occur, as illustrated in Fig. 6 for a $D_{x}=1 \mathrm{~cm}$ horizontal dispersion at the electron lens.

To investigate the effect of spurious dispersion at the electron lens on the attainable luminosity, we performed tune scans for different values of the dispersion $D_{x}=D_{y}$ at the electron lens. Figure 7 shows the resulting maximum normalized luminosity $L_{\text {norm }}$ over the range $0 \leq D_{x}, D_{y} \leq$ $2 \mathrm{~cm}$.

\section{B. Transverse offsets}

Transverse beam offsets at the IP and/or the electron lens give rise to odd-order resonances, in addition to even-order resonances that are driven by the head-on beam-beam interaction. While careful steering of the beams in collision is used to minimize these offsets, residual imperfections

TABLE II. Normalized luminosity $L_{\text {norm }}$ in units of $10^{11}$ as a function of the size ratios $r_{x}=\sigma_{x, e} / \sigma_{x, p}$, and $r_{y}=\sigma_{y, e} / \sigma_{y, p}$. The subscript " $e$ " refers to the electron lens beam, while the subscript " $p$ " corresponds to the proton beam. Since we deliberately chose the same beta functions at both the IP and the electron lens, equal beam sizes $\sigma_{x, e}=\sigma_{x, p}, \sigma_{y, e}=\sigma_{y, p}$ and intensities $N_{e}=N_{p}$ correspond to the case of full beam-beam compensation.

\begin{tabular}{|c|c|c|c|c|c|c|c|}
\hline & \multicolumn{6}{|c|}{$r_{x}$} \\
\hline & & 1.00 & 1.05 & 1.10 & 1.15 & 1.20 & 1.25 \\
\hline \multirow[t]{6}{*}{$r_{y}$} & 1.00 & $2.79 \pm 0.02$ & $2.87 \pm 0.02$ & $2.96 \pm 0.02$ & $3.04 \pm 0.02$ & $3.07 \pm 0.02$ & $3.11 \pm 0.07$ \\
\hline & 1.05 & $2.85 \pm 0.03$ & $2.95 \pm 0.03$ & $3.01 \pm 0.01$ & $3.02 \pm 0.03$ & $3.09 \pm 0.03$ & $3.12 \pm 0.06$ \\
\hline & 1.10 & $2.97 \pm 0.02$ & $2.99 \pm 0.05$ & $3.03 \pm 0.05$ & $3.08 \pm 0.05$ & $2.99 \pm 0.02$ & $3.06 \pm 0.05$ \\
\hline & 1.15 & $2.97 \pm 0.06$ & $3.01 \pm 0.06$ & $3.05 \pm 0.05$ & $3.05 \pm 0.03$ & $3.06 \pm 0.07$ & $3.05 \pm 0.06$ \\
\hline & 1.20 & $2.96 \pm 0.09$ & $3.00 \pm 0.08$ & $2.99 \pm 0.08$ & $3.07 \pm 0.19$ & $3.00 \pm 0.13$ & $3.04 \pm 0.03$ \\
\hline & 1.25 & $2.93 \pm 0.10$ & $3.00 \pm 0.09$ & $3.02 \pm 0.11$ & $2.92 \pm 0.14$ & $3.05 \pm 0.14$ & $2.95 \pm 0.04$ \\
\hline
\end{tabular}

TABLE III. Normalized luminosity $L_{\text {norm }}$ in units of $10^{11}$ as a function of intensity ratio $N_{e} / N_{p}$ and the beam size ratio $r=r_{x}=$ $\sigma_{x, e} / \sigma_{x, p}=r_{y}=\sigma_{y, e} / \sigma_{y, p}$. The subscript " $e$ " refers to the electron lens beam, while the subscript " $p$ " corresponds to the proton beam. Since we deliberately chose the same beta-functions at both the IP and the electron lens, equal beam sizes $\sigma_{x, e}=\sigma_{x, p}, \sigma_{y, e}=$ $\sigma_{y, p}$ and intensities $N_{e}=N_{p}$ correspond to the case of full beam-beam compensation.

\begin{tabular}{|c|c|c|c|c|c|c|}
\hline & & 0.80 & 0.85 & $\begin{array}{c}N_{e} / N_{p} \\
0.90\end{array}$ & 0.95 & 1.00 \\
\hline \multirow[t]{6}{*}{$r$} & 1.00 & $3.55 \pm 0.06$ & $3.37 \pm 0.06$ & $3.18 \pm 0.01$ & $3.01 \pm 0.03$ & $2.79 \pm 0.02$ \\
\hline & 1.05 & $3.56 \pm 0.10$ & $3.42 \pm 0.04$ & $3.34 \pm 0.07$ & $3.16 \pm 0.04$ & $2.95 \pm 0.03$ \\
\hline & 1.10 & $3.48 \pm 0.16$ & $3.36 \pm 0.03$ & $3.36 \pm 0.10$ & $3.19 \pm 0.09$ & $3.03 \pm 0.05$ \\
\hline & 1.15 & $3.41 \pm 0.23$ & $3.30 \pm 0.07$ & $3.31 \pm 0.09$ & $3.18 \pm 0.18$ & $3.05 \pm 0.03$ \\
\hline & 1.20 & $3.23 \pm 0.16$ & $3.15 \pm 0.15$ & $3.15 \pm 0.10$ & $3.17 \pm 0.24$ & $3.00 \pm 0.13$ \\
\hline & 1.25 & $3.06 \pm 0.24$ & $3.06 \pm 0.13$ & $3.00 \pm 0.14$ & $2.99 \pm 0.07$ & $2.95 \pm 0.04$ \\
\hline
\end{tabular}




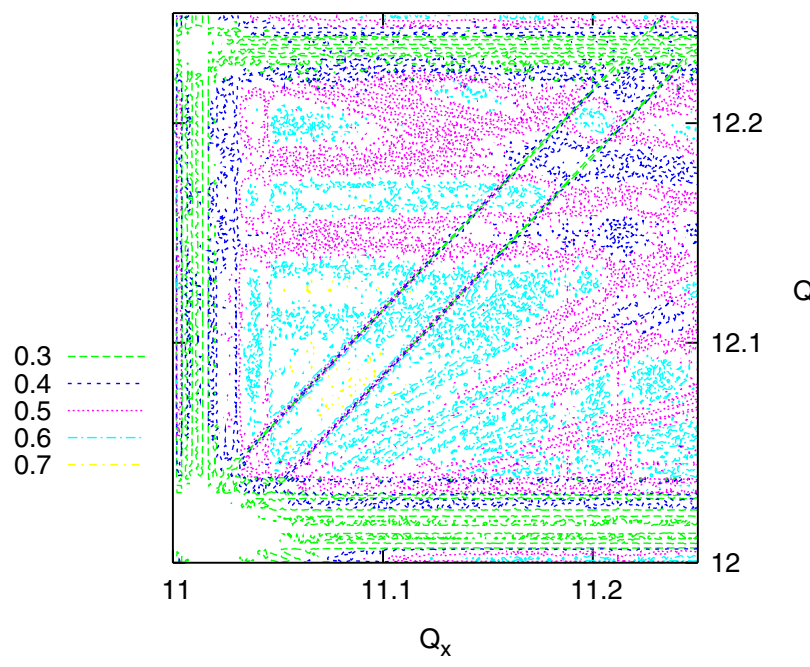

FIG. 6. (Color) Tune scan for a spurious horizontal dispersion of $D_{x}=1 \mathrm{~cm}$ at the electron lens. This tune scan is performed on a grid of $\Delta Q_{x, y}=0.001$ to reveal synchrobetatron resonances at a synchrotron tune of $Q_{s}=0.015$.

are practically unavoidable. It is therefore important to ensure a sufficient robustness of the beam-beam compensation scheme against such residual beam-beam offsets.

Figure 8 shows the maximum luminosity $L_{\text {norm }}$ for different offsets $\delta x / \sigma_{x}=\delta_{y} / \sigma_{y}$, over a range between 0 and 0.5 .

\section{Phase advance between IP and electron lens}

In the ideal case of a perfectly linear transport channel between the IP and the electron lens, complete compensation of the beam-beam effect would occur if the betatron phase advance in both transverse planes was an exact multiple of $180^{\circ}$. To study the dependence of the maximum attainable luminosity on this betatron phase advance in our

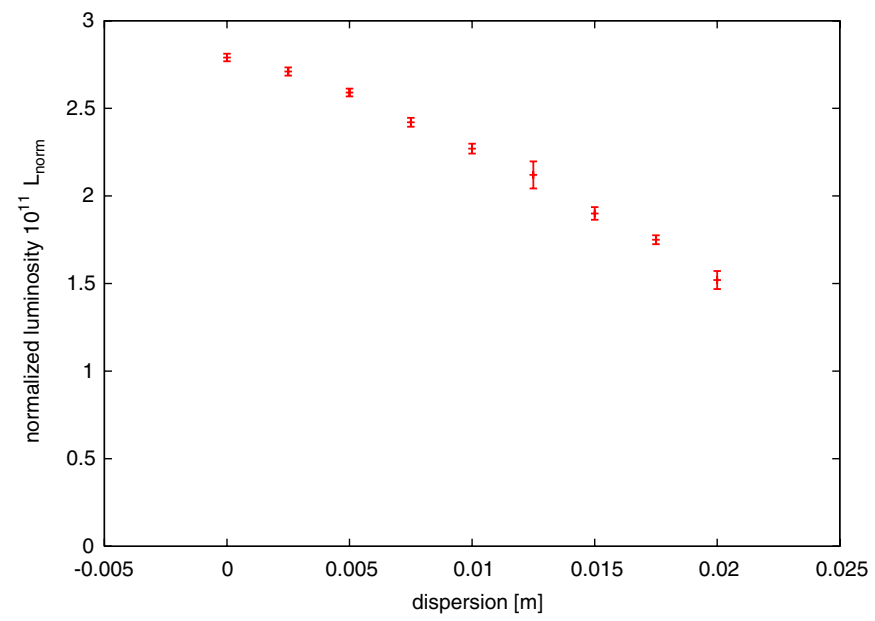

FIG. 7. (Color) Maximum normalized luminosity obtained during tune scans as a function of the spurious dispersion $D_{x}=D_{y}$ at the electron lens.

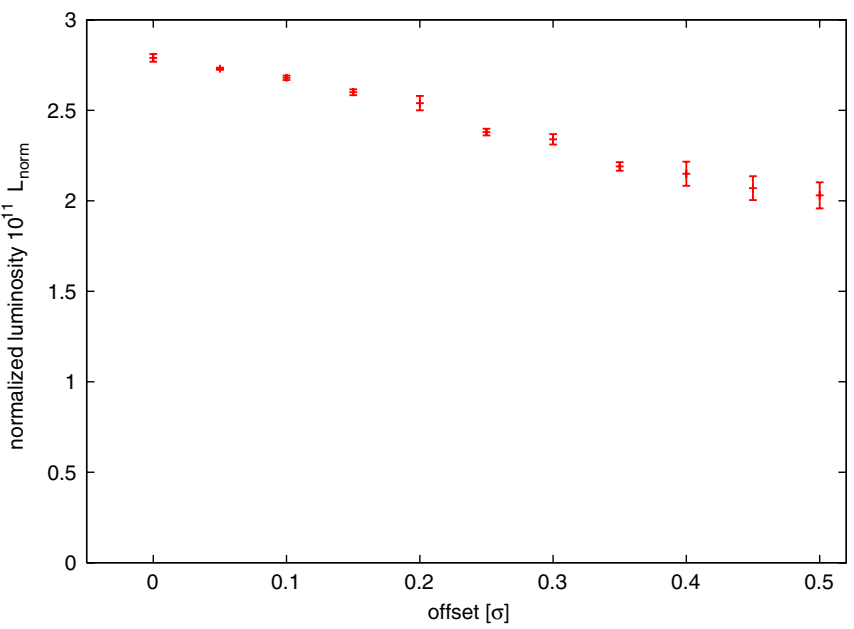

FIG. 8. (Color) Maximum normalized luminosity obtained during tune scans as a function of the relative beam offset $\delta x / \sigma_{x}=$ $\delta_{y} / \sigma_{y}$ at the electron lens.

nonlinear electron ring model lattice, the phase advance has been varied in steps of $1.8^{\circ}$ in both planes. As Fig. 9 shows, the maximum luminosity drops significantly if the phase error exceeds about $4^{\circ}$. Also note that for negative phase advance errors the maximum luminosity occurs at working point $(0.21,0.22)$, very different from the best working point for positive phase errors $(0.04,0.06)$.

\section{Intensity mismatch}

Since the intensity of the corresponding proton bunch can vary considerably along the bunch train, it is important to ensure that the beam-beam compensation scheme works successfully despite an intensity mismatch between proton bunch and electron lens beam. As Fig. 10 indicates, larger luminosities can be gained with undercompensation

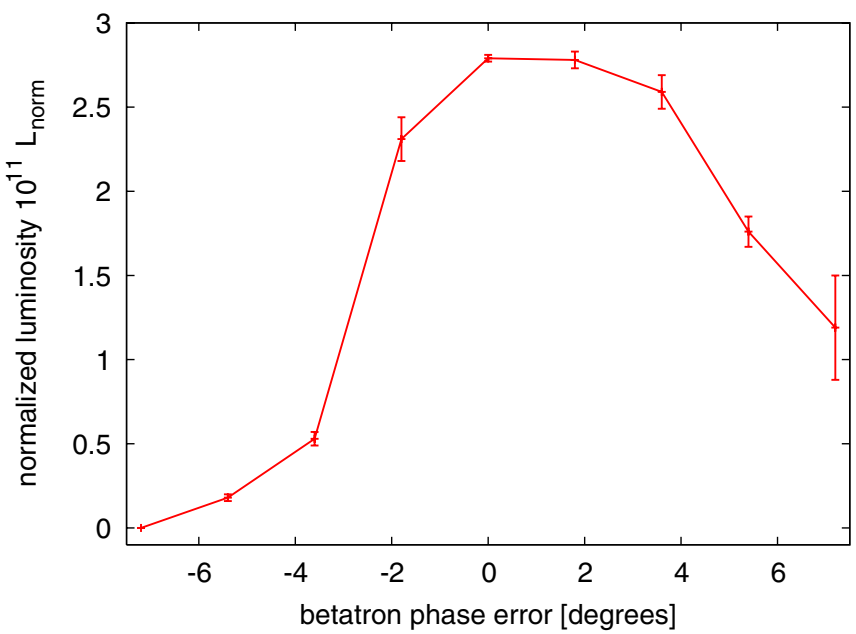

FIG. 9. (Color) Maximum normalized luminosity obtained during tune scans as a function of the betatron phase error between IP and electron lens. 


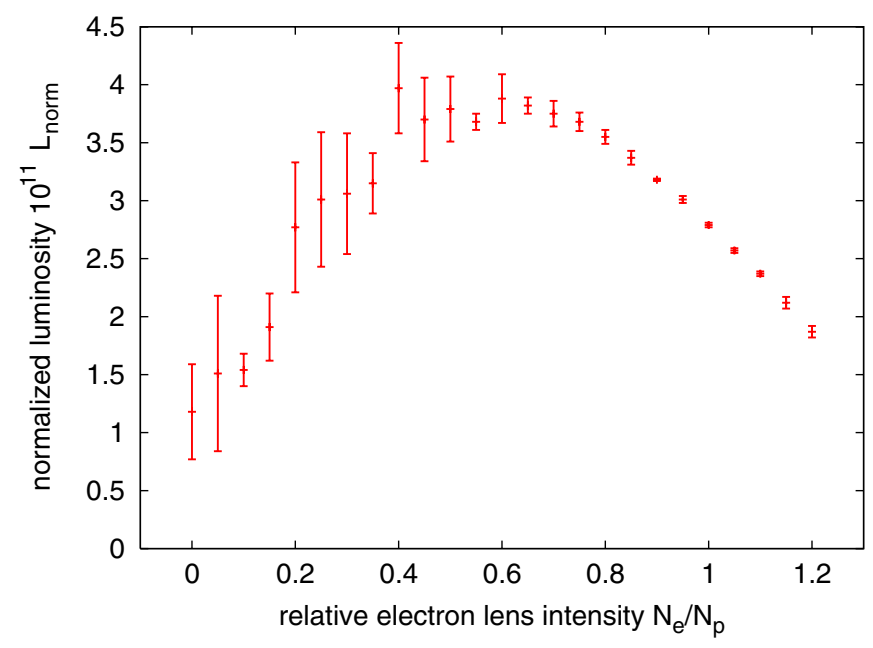

FIG. 10. (Color) Maximum normalized luminosity obtained during tune scans as a function of the relative intensity $N_{e} / N_{p}$ of the electron lens. While in the undercompensated case $N_{e} / N_{p}<1$ the maximum luminosity occurs at fractional tunes of $\left(Q_{x}, Q_{y}\right)=(0.04,0.06)$, the highest luminosity in the overcompensated case $N_{e} / N_{p}>1$ is reached at a working point near $(0.20,0.21)$.

$N_{e}<N_{p}$ and a maximum is reached around $N_{e} / N_{p}=0.5$ Overcompensation, $N_{e}>N_{p}$, reduces the luminosity. The tune space where these high luminosities can be achieved shrinks with the intensity of the electron lens beam $N_{e}$, as Fig. 3 shows, until it eventually becomes a single point in the uncompensated case $N_{e}=0$.

Moreover, the optimum working point in the overcompensated case differs significantly from the one in the undercompensated case. If a DC beam is used in the electron lens it is therefore necessary to choose the electron lens beam intensity such that no overcompensation occurs for any bunch despite the bunch-to-bunch intensity variations of the proton beam.

\section{E. Beam size mismatch}

Besides the bunch intensity, the rms transverse beam sizes $\sigma_{x, p}$ and $\sigma_{y, p}$ of the proton beam can easily vary by $10 \%$ to $20 \%$ from bunch to bunch. Since these variations are not necessarily correlated in the two transverse planes, we varied them independently in both planes. Table II lists the maximum luminosity for different values of the transverse beam size ratios $r_{x}=\sigma_{x, e} / \sigma_{x, p}$ and $r_{y}=\sigma_{y, e} / \sigma_{y, p}$. A beam size mismatch of $10 \%$ in both planes reduces the maximum luminosity by about the same amount.

As already observed in the case of intensity mismatch between proton bunch and electron lens intensity, overcompensation with $r_{x}<1$ or $r_{y}<1$ results in the maximum luminosity occurring at a working point different from the undercompensated cases where the maximum luminosity occurs around $(0.04,0.06)$.

\section{F. Simultaneous intensity and size mismatch}

Finally, we need to ensure that simultaneous mismatches of both beam size and intensity between proton beam and electron lens provide sufficient luminosity. Table III shows the resulting luminosity for different values of the intensity ratio $N_{e} / N_{p}$ and the beam size ratio $r=\sigma_{e} / \sigma_{p}$.

\section{G. Nonlinearities between the interaction point and the electron lens}

In a perfectly linear lattice, and in the absence of quantum excitation and radiation damping between IP and electron lens, perfect compensation of the nonlinear beam-beam effect can be achieved. However, this idealized case is unrealistic. To investigate the effect of nonlinear elements (sextupoles) on the beam-beam compensation, we have varied the number $N_{\text {sep }}$ of FODO cells, and therefore the number of sextupoles, between the IP and the electron lens, while the betatron phase advances between IP and electron lens are adjusted to be integer multiples of $180^{\circ}$. Together with $N_{\text {sep }}$, the amount of synchrotron radiation has been varied accordingly. Figure 11 shows the maximum achieved luminosity in the tune region $0<Q_{x}$, $Q_{y}<0.25$ for three different numbers of FODO cells between the IP and the electron lens, together with the uncompensated case, as a function of the proton bunch intensity $N_{p}$.

The three cases show that with an increase in the nonlinearities, the beam-beam parameter above which the luminosity drops is reduced. However, for the nonlinearities tested, beam-beam parameters larger than 0.4 are needed to see a difference.

In the case without beam-beam compensation, the emittance growth due to the beam-beam interaction is over-

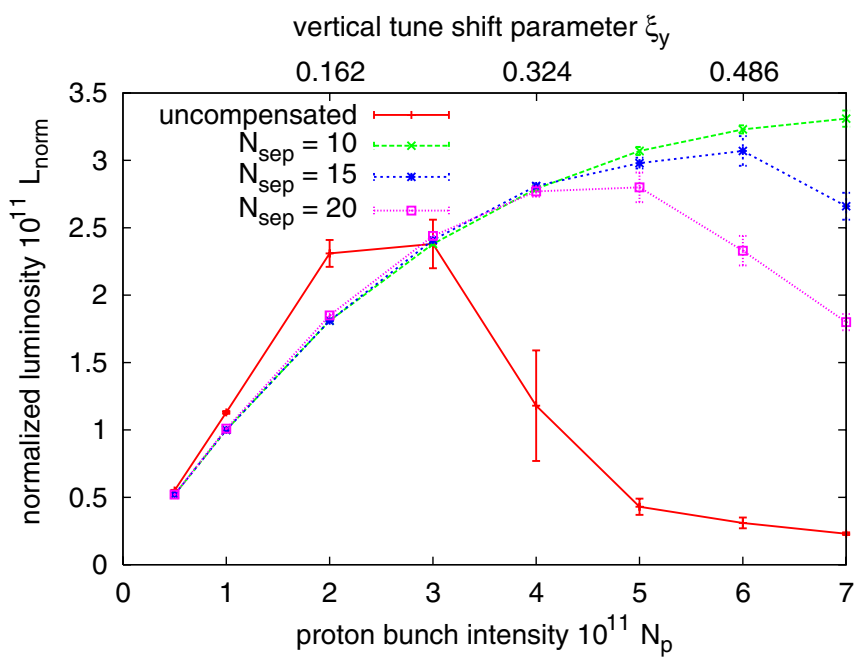

FIG. 11. (Color) Maximum achieved normalized luminosity $L_{\text {norm }}$ for three different numbers of FODO cells $N_{\text {sep }}$, separating the IP and the electron lens. The uncompensated case (no electron lens) is shown for comparison. 
compensated by the dynamic focusing effect at the IP for proton bunch intensities $N_{p}$ up to $2 \times 10^{11}$, resulting in a net luminosity enhancement due to electron beam sizes smaller than the design value. Beyond $N_{p}=3 \times 10^{11}$ emittance growth overwhelms the dynamic focusing, causing the luminosity to drop rapidly when the proton bunch intensity is further increased.

With full head-on beam-beam compensation, the lack of dynamic focusing results in a slight luminosity reduction due to some residual emittance blowup. However, depending on the number of FODO cells $N_{\text {sep }}$ between the IP and the electron lens, the normalized luminosity $L_{\text {norm }}$ still increases with the proton bunch intensity $N_{p}$ until a maximum is reached, though the increase is less than linear. Note that while the beam core size is smaller in the uncompensated case for proton bunch intensities up to $3 \times$ $10^{11}$, the non-Gaussian tails are more pronounced (see Fig. 5) and may already prevent operation at these intensities.

\section{MAXIMIZING THE LUMINOSITY}

In the previous section we found that partial compensation with $N_{e}<N_{p}$ generally results in higher luminosities than full compensation $\left(N_{e}=N_{p}\right)$. We can use this result to maximize the luminosity. As Fig. 10 shows, the maximum luminosity for the proton intensity of $N_{p}=4 \times 10^{11}$ is reached at approximately half compensation, $N_{e} / N_{p}=$ 0.5 . Figure 12 shows the maximum normalized luminosity $L_{\text {norm }}$ as a function of the proton beam intensity $N_{p}$, for half compensation $N_{e} / N_{p}=0.5$. With half compensation, the luminosity with the electron lens is never smaller than the luminosity without the compensation, and the proton in-

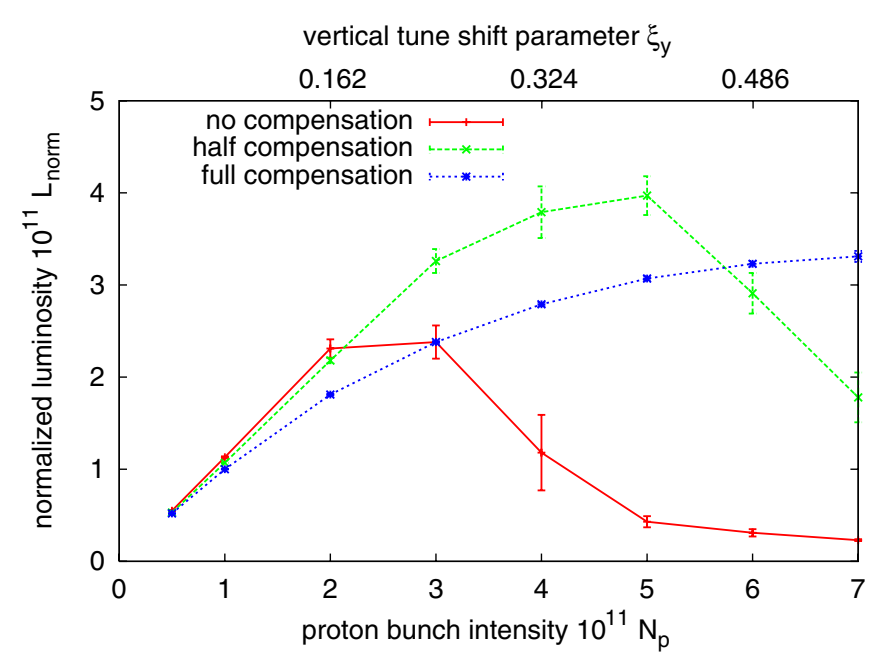

FIG. 12. (Color) Maximum normalized luminosity obtained during tune scans as a function of the proton bunch intensity $N_{p}$, for half compensation $N_{e} / N_{p}=0.5$. The cases for zero $\left(N_{e} / N_{p}=\right.$ $0)$ and full $\left(N_{e} / N_{p}=1.0\right)$ compensation are shown for comparison, and are identical to those shown in Fig. 11. tensity beyond which the luminosity decreases is approximately doubled.

The electron lens also helps when the luminosity is limited by the formation of non-Gaussian tails below the proton intensity where it saturates. Figure 5 shows that with half compensation, the proton intensity could be doubled from $1 \times 10^{11}$ to $2 \times 10^{11}$, or from $2 \times 10^{11}$ to $4 \times 10^{11}$ while maintaining the size of the tails.

We conclude that use of an electron lens permits us to approximately double the proton intensity and thereby the luminosity for both the first and second beam-beam limits.

\section{SUMMARY}

We performed weak-strong beam-beam simulations to investigate the feasibility of head-on beam-beam compensation of an electron beam in an electron-ion collider. In the parameter regime tested, the simulations show that the beam-beam parameter can be doubled for both the first (beam core size) and second (non-Gaussian transverse tails) beam-beam limit. The electron lens also reduces the sensitivity of the luminosity to tune changes, which offers more flexibility if the spin polarization needs to be maintained in the electron beam.

We found that the best compensation is obtained with about half the beam-beam strength in the interaction point. At this strength the compensation is robust against further intensity mismatch, as it is against beam size mismatch. The compensation is, however, rather sensitive to phase deviations from the optimum value of multiples of $180^{\circ}$. We found that these errors have to be controlled to within $\pm 2^{\circ}$.

\section{ACKNOWLEDGMENTS}

We are thankful for discussions of beam dynamics and simulation problems with N. Abreu, M. Blaskiewicz, Y. Luo, G. Robert-Demolaize, H.-J. Kim, T. Sen, R. Talman, and A. Valishev. We are also thankful to V. Kamerdzhiev, V. Shiltsev, and A. Valishev for the insight they provided into the operation of electron lenses in the Tevatron. T. Satogata's help with proofreading the manuscript is gratefully acknowledged.

[1] L. Ahrens et al., BNL Note C-A/AP/142.

[2] R. Talman (private communication).

[3] G. Arzelia, L. Belbéoch, M. Bergher, P. Brunet, X. Buffet, R. Chehab, S. Costa, G. Danon, J. Dupin, S. Getka, A. Jejcic, J. LeDuff, M. P. Level, R. Liot, P. Marin, C. Nguyen Ngoc, D. Potaux, M. Sommer, R. Souchet, and H. Zyngier, in Proceedings of the 8th International Conference on High Energy Accelerators, Geneva, 1971, p. 150.

[4] Y. S. Derbenev, 3rd all Union Conference on Accelerators, 1972; INP 70-72, 1972; SLAC TRANS 151, 1973.

[5] E. Keil, in Proceedings of the 3rd ICFA Beam Dynamics Workshop, 1989; CERN-LEP-TH/89-37, 1989. 
[6] E. Tsyganov, R. Meinke, W. Nexsen, and A. Zinchenko, SSCL-PREPRINT-519, 1993.

[7] E. Tsyganov, A. Taratin, and A. Zinchenko, JINR-E9-964, 1996.

[8] V. Shiltsev and A. Zinchenko, Phys. Rev. ST Accel. Beams 1, 064001 (1998).

[9] A. Burov, V. Danilov, and V. Shiltsev, Phys. Rev. E 59, 3605 (1999).

[10] V. Shiltsev, V. Danilov, D. Finley, and A. Sery, Phys. Rev. ST Accel. Beams 2, 071001 (1999).

[11] V. Shiltsev, Y. Alexahin, V. Kamerdzhiev, G. Kuznetsov, X. Zhang, and K. Bishofberger, Phys. Rev. Lett. 99, 244801 (2007).

[12] V. Shiltsev, Y. Alexahin, K. Bishofberger, V. Kamerdzhiev, V. Parkhomchuk, V. Reva, N. Solyak, D. Wildman, X. Zhang, and F. Zimmermann, New J. Phys. 10, 043042 (2008).

[13] Y. Luo, N.P. Abreu, W. Fischer, and G. RobertDemolaize, in Proceedings of the 11th European Particle Accelerator Conference, Genoa, 2008 (EPS-AG, Genoa, Italy, 2008).

[14] N. Abreu, W. Fischer, Y. Luo, and G. Robert-Demolaize, in Proceedings of the 11th European Particle Accelerator Conference, Genoa, 2008 (Ref. [13]).

[15] U. Dorda, F. Zimmermann, W. Fischer, and V. Shiltsev, in Proceedings of the 2007 Particle Accelerator Conference,
Albuquerque, New Mexico, 2007 (IEEE, Albuquerque, New Mexico, 2007), pp. 1589-1591; LHC-PROJECTReport-1023, 2007.

[16] B. Podobedov and R. H. Siemann, Phys. Rev. E 52, 3066 (1995).

[17] Y. Ohnishi and K. Ohmi, in Proceedings of HALO'03 and Beam-Beam'03, Montauk, New York, AIP Conf. Proc. No. 693 (AIP, New York, 2003).

[18] J. Shi and D. Yao, Phys. Rev. E 62, 1258 (2000).

[19] L. Jin and J. Shi, Phys. Rev. E 69, 036503 (2004).

[20] Y. Alexahin, in Proceedings of the 21st Particle Accelerator Conference, Knoxville, 2005 (IEEE, Piscataway, NJ, 2005).

[21] W. Fischer et al., in Proceedings of the 20th Particle Accelerator Conference, Portland, OR, 2003 (IEEE, New York, 2003).

[22] J. Shi, L. Jin, and F. Wang, Nucl. Instrum. Methods Phys. Res., Sect. A 555, 6 (2005).

[23] M. Bassetti and G. Erskine, CERN Report No. ISR TH/8006, 1980.

[24] R.H. Siemann, SLAC Report No. SLAC-PUB-6073, 1993.

[25] J. T. Seeman, SLAC Report No. SLAC-PUB-3182, 1983.

[26] D. Shatilov, Part. Accel. 52, 65 (1996).

[27] T. Chen, J. Irwin, and R. Siemann, Phys. Rev. E 49, 2323 (1994). 